\title{
Primordial black holes from fifth forces
}

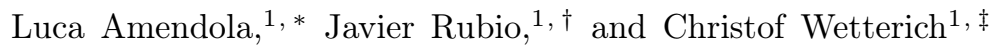 \\ ${ }^{1}$ Institut für Theoretische Physik, Ruprecht-Karls-Universität Heidelberg, \\ Philosophenweg 16, 69120 Heidelberg, Germany
}

\begin{abstract}
Primordial black holes can be produced by a long range attractive fifth force stronger than gravity, mediated by a light scalar field interacting with nonrelativistic "heavy" particles. As soon as the energy fraction of heavy particles reaches a threshold, the fluctuations rapidly become nonlinear. The overdensities collapse into black holes or similar screened objects, without the need for any particular feature in the spectrum of primordial density fluctuations generated during inflation. We discuss whether such primordial black holes can constitute the total dark matter component in the Universe.
\end{abstract}

\section{INTRODUCTION}

In spite of the many observations leading to the establishment of dark matter as an essential ingredient of modern cosmology, its fundamental nature remains an open question. Among the many dark matter candidates, primordial black holes $(\mathrm{BH})$ [14 are interesting since they could account for the gravitational wave signals observed by the Laser Interferometer Gravitational Wave Observatory (LIGO) and the VIRGO observatory [5] or seed the formation of supermassive black holes [6] (see also [7] and references therein). The existence of primordial BHs could be a natural consequence of inflation. In particular, if the inflationary potential contains a nontrivial feature along the inflaton trajectory, the spectrum of primordial perturbations might develop a peak at intermediate scales. If the amplitude of this peak is large enough, the nonlinear perturbations will collapse into BHs after horizon reentry 8]. Alternatively, the formation of primordial BHs could take place at phase transitions [9] or be associated with the fragmentation of a scalar condensate into Q-balls [10].

In this paper we present a novel framework for primordial BH formation which does not rely on a particular feature in the spectrum of primordial density fluctuations generated during inflation. The main assumption of our scenario is the presence in the early Universe of a longrange interaction stronger than gravity. We associate this fifth force to a light scalar field interacting with some heavy degrees of freedom beyond the Standard Model particle content. More precisely, we assume that during some epoch in cosmology the Hubble parameter $H$ is larger than the mass of a scalar field $\phi$. If this scalar field couples to some "heavy particles" $\psi$ with masses larger than $H$, it mediates an attractive fifth force which is effectively long range, similar to gravity. This attraction can be, however, substantially stronger than the gravitational attraction. As a result, the fluctuations in the energy density of the heavy fields can grow rapidly and eventually become nonlinear. If the range and strength of the fifth force is large enough, it seems likely that a substantial part of the $\psi$ fluid will collapse into BHs or similar screened objects.

The existence of long-range attractive forces stronger than gravity is a natural expectation in particle physics models containing scalar fields. The simplest example is the attractive interaction among Standard Model fermions via Higgs particle exchange. In early cosmology it is much stronger than the gravitational attraction. If not countered by electromagnetic interactions, even the Standard Model Higgs would have induced gravitational collapse at early times. Alternatively, the $\phi$ and $\psi$ fields could be associated with grand unified frameworks involving, for instance, a scalar triplet interacting with heavy neutrinos 11. In this case, the $\mathrm{BH}$ formation process could occur very early in cosmology, for example nearly after the end of inflation. For different properties of the participating particles it could also take place rather late in the cosmological history, say after nucleosynthesis. The heavy particles remaining outside primordial BHs might decay after the formation epoch and be unobservable today. The scalar field could relax after $\mathrm{BH}$ formation to a minimum of its effective potential with mass eventually exceeding the decreasing Hubble parameter. In this case the field $\phi$ would not be observable at the present time either. Alternatively, $\phi$ could be an additional dark matter candidate, or have a runaway behavior and be associated with dynamical dark energy. The BH formation process is not affected by what happens to the participating fields or particles at later times. Once BHs are formed, they behave as nonrelativistic matter. If the total energy density of BHs is large enough, they could constitute the dark matter component of our Universe.

\section{FIFTH-FORCE INTERACTIONS}

Consider the action

$$
S=\int d^{4} x \sqrt{-g}\left[\frac{M_{P}^{2}}{2} R+\mathcal{L}_{R}+\mathcal{L}(\phi)+\mathcal{L}(\phi, \psi)\right],
$$

with $M_{P}=(8 \pi G)^{-1 / 2}=2.435 \times 10^{18} \mathrm{GeV}$ the reduced Planck mass and $\mathcal{L}_{R}$ a radiation component that we assume to dominate the background evolution during the 
epoch relevant for primordial $\mathrm{BH}$ formation. The term

$$
\mathcal{L}(\phi)=-\frac{1}{2} \partial^{\mu} \phi \partial_{\mu} \phi-V(\phi),
$$

stands for the Lagrangian density of an almost massless scalar field $\phi$. We will assume for simplicity that the potential $V(\phi)$ can be neglected during the epoch of $\mathrm{BH}$ formation such that $\phi$ becomes effectively a massless field. This approximation is justified if the scalar field mass is smaller than $H$, both for a potential with a minimum or for a runaway potential. For definiteness we take the heavy particle $\psi$ to be a fermion. The interactions with the field $\phi$ arise via a field-dependent mass term $m(\phi)$,

$$
\mathcal{L}(\phi, \psi)=i \bar{\psi}\left(\gamma^{\mu} \nabla_{\mu}-m(\phi)\right) \psi,
$$

for example by a Yukawa type coupling $\sim g \phi \bar{\psi} \psi$.

We will assume that the field equations derived from the action (1) admit a perfect fluid description and consider a flat Friedmann-Lemaître-Robertson-Walker Universe. The background evolution equations for the average $\phi$ and $\psi$ energy densities read

$$
\begin{aligned}
& \dot{\rho}_{\phi}+3 H\left(\rho_{\phi}+p_{\phi}\right)=\frac{\beta}{M_{P}}\left(\rho_{\psi}-3 p_{\psi}\right) \dot{\phi}, \\
& \dot{\rho}_{\psi}+3 H\left(\rho_{\psi}+p_{\psi}\right)=-\frac{\beta}{M_{P}}\left(\rho_{\psi}-3 p_{\psi}\right) \dot{\phi},
\end{aligned}
$$

with $\rho_{\phi}=p_{\phi}=\dot{\phi}^{2} / 2, H^{2}=\rho /\left(3 M_{P}^{2}\right)$ and $\rho=\rho_{R}+$ $\rho_{\phi}+\rho_{\psi}$. The $\phi$ and $\psi$ fluids are coupled whenever the $\psi$ particles are nonrelativistic $\left(\rho_{\psi} \neq 3 p_{\psi}\right.$, with $p_{\psi}$ the $\psi$-fluid pressure). The coupling function $\beta(\phi)$ measures the dependence of the effective mass $m(\phi)$ on the scalar field $\phi$,

$$
\beta(\phi)=-M_{P} \frac{\partial \ln m(\phi)}{\partial \phi} .
$$

Its normalization involving $M_{P}$ has been chosen such that for $\beta^{2}=1 / 2$ the scalar-field mediated attraction has the same strength as gravity. The combined strength of the fifth force and gravity is proportional to

$$
Y \equiv 1+2 \beta^{2} .
$$

This type of scenario has been extensively studied in the literature [12 17], but not within the context of primordial black hole formation. A given model is specified by a choice of $\beta(\phi)$. The value of $\beta$ can be rather large. Consider, for instance, a renormalizable interaction term of the form $m(\phi) \bar{\psi} \psi=m_{0} \bar{\psi} \psi+g \phi \bar{\psi} \psi$ with $m_{0}$ a constant mass parameter and $g$ a dimensionless coupling. For this particular example, we can rewrite Eq. (6) as $\beta(\phi)=-g M_{P} / m(\phi)$, which leads to $|\beta| \gg 1$ if $m(\phi) / M_{P} \ll g$. Even a small value of the Yukawa coupling $g$ can be largely overwhelmed by the ratio $M_{P} / m$. The factor $2 \beta^{2}$ can therefore be naturally rather large.

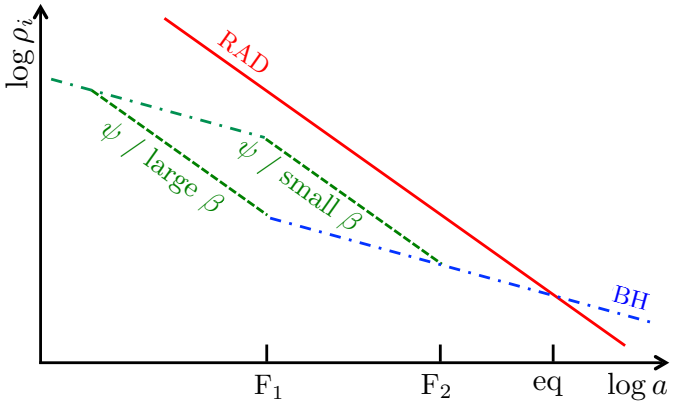

Figure 1. Schematic evolution of the energy density of different species. The red and blue lines stand respectively for radiation and $\mathrm{BH}$ dark matter. Green lines denote $\rho_{\psi}$. Larger values of $\beta$ imply earlier $\mathrm{BH}$ formation and therefore smaller masses. For $\Omega_{\mathrm{BH}}\left(a_{\mathrm{eq}}\right)<1 / 2$ the radiation line moves upwards.

For illustration purposes we will neglect the field dependence of $\beta$ in the following sections. The qualitative features of the BH formation scenario presented here do not rely on this approximation. They also hold if we take for $\psi$ a nonrelativistic bosonic particle rather than a fermion.

\section{BACKGROUND COSMOLOGY}

For a nonrelativistic fluid of $\psi$ particles, $p_{\psi}=0$, the set of equations (4)-(5) admits a scaling solution. In the limit $\beta \gg 1$ it reads [18]

$$
\Omega_{\psi}=\frac{1}{3 \beta^{2}}, \quad \Omega_{\phi}=\frac{1}{6 \beta^{2}}, \quad \Omega_{R}=1-\frac{1}{2 \beta^{2}},
$$

where $\Omega_{i} \equiv \rho_{i} /\left(3 M_{P}^{2} H^{2}\right)$ and $i=R, \phi, \psi$. During this scaling phase, the fermion energy density tracks the background component, $\rho_{\psi} \sim \rho_{R} \sim a^{-4}$. Combining this scaling with the intuitive solution of Eq. (5), $\rho_{\psi}=\rho_{\psi, 0} a^{-3} \exp \left(-\beta \phi / M_{P}\right)$, we obtain a relation between $\beta$ and the variation of the $\phi$ field during the scaling phase, namely

$$
\phi^{\prime}=M_{P} / \beta,
$$

with primes denoting derivatives with respect to the number of $e$-folds $d N \equiv d \ln a$, e.g. $\phi^{\prime}=\dot{\phi} / H$.

Alternatively, $\Omega_{\psi}$ could be even smaller than $1 /\left(3 \beta^{2}\right)$. The fifth force then plays no role for the background evolution and $\rho_{\psi}$ decays as nonrelativistic matter, $\rho_{\psi} \sim a^{-3}$. In consequence, the density parameter $\Omega_{\psi}$ increases with time until it reaches the scaling solution $\Omega_{\psi}=1 /\left(3 \beta^{2}\right)$ at some time $t_{\text {in }}$. The evolution of the different energy densities is depicted in Fig. 1 .

Which of the above regimes is realized during the $\mathrm{BH}$ formation period depends on the initial conditions for the energy densities $\rho_{\psi}$ and $\rho_{\phi}$, which are typically set 
at the end of inflation. For the large $\beta^{2}$ values we will be interested in, both the difference between various initial conditions and the question of whether or not the scaling solution (8) is reached become unimportant from the point of view of the background evolution. For all practical purposes, the background behaves as a standard radiation-dominated (RD) Universe with a Hubble parameter obeying $\mathcal{H}^{\prime}=-\mathcal{H}$, with $\mathcal{H}=a H$.

\section{GROWTH OF FLUCTUATIONS}

Due to the strong attractive force for $Y \gg 1$ and the decreasing particle masses, the $\psi$ fluctuations grow rapidly for sufficiently large $\Omega_{\psi}$. On scales $k^{-1}$ sufficiently inside the horizon, the linear perturbations $\delta_{\psi}$ in the $\psi$ fluid evolve as 19

$$
\delta_{\psi}^{\prime \prime}+\left(1+\frac{\mathcal{H}^{\prime}}{\mathcal{H}}-\frac{\beta \phi^{\prime}}{M_{P}}\right) \delta_{\psi}^{\prime}-\frac{3}{2}\left(Y \Omega_{\psi} \delta_{\psi}+\Omega_{R} \delta_{R}\right)=0 .
$$

For large $\beta^{2}$ the large value of $Y$ accounts for the enhanced attraction between the $\psi$ particles. In addition we observe a generalized damping (or rather antidamping) term $\sim \beta \phi^{\prime}$ due to the change of the heavy particle mass as $\phi$ evolves. The perturbations in $\rho_{\phi}$ are negligible in view of a unit sound speed. The perturbations $\delta_{R}$ in the radiation fluid follow the standard behavior and do not grow. Their small amplitude remains at the level inherited from inflation.

Even for a very small initial perturbation $\delta_{\psi}$ the inhomogeneities in the radiation fluid will trigger inhomogeneities in the $\psi$ fluid due to the source term $\delta_{R}$ in the evolution equation for $\delta_{\psi}$. This typically implies a minimal value for $\delta_{\psi}$ of the same order as $\delta_{R}$. For the further growth of $\delta_{\psi}$ we can neglect $\delta_{R}$.

We first discuss the growth rate for the scaling solution (8). Inserting Eq. (9), together with $Y \Omega_{\psi} \simeq 2 / 3$ and $\mathcal{H}^{\prime}=-\mathcal{H}$, and neglecting $\delta_{R}$, the evolution equation 10 . becomes rather simple,

$$
\delta_{\psi}^{\prime \prime}-\delta_{\psi}^{\prime}-\delta_{\psi}=0 .
$$

The solution of this differential equation contains a growing and a decaying mode. At large $a$, we are left only with the growing piece, namely

$$
\delta_{\psi}=\delta_{\psi, \text { in }}\left(a / a_{\text {in }}\right)^{p}, \quad p=(1+\sqrt{5}) / 2 \approx 1.62,
$$

with $a_{\text {in }} \equiv a\left(t_{\text {in }}\right)$ the scale factor at the onset of the scaling regime. The $\phi-\psi$ interactions in Eqs. (4) and (5) translate into a power-law growth of the $\psi$ perturbations during the scaling regime. This is a key difference with respect to nonrelativistic matter without the fifth force. For $\beta=0$ the fluctuations $\delta_{\psi}$ do not grow during radiation domination.

The scaling solution is not essential for the growth of $\psi$ perturbations. For $\Omega_{\psi}>1 /\left(3 \beta^{2}\right)$ the growth is faster than for the scaling solution, while for $\Omega_{\psi}<1 /\left(3 \beta^{2}\right)$ the growth slows down. The growth also slows if $\phi$ changes slower than the scaling solution (9). Furthermore, the growth rate decreases as the wavelength of fluctuations increases towards the horizon. Many scenarios can be covered by treating $p$ as approximately constant and roughly of the order one. For constant $p$ even small initial inhomogeneities $\delta_{\psi}$, e.g. $\delta_{\psi \text {,in }} \sim 10^{-5}$, will develop into nonlinear inhomogeneities rather rapidly. The number of $e$-folds for the onset of nonlinearity is

$$
N_{\mathrm{F}} \equiv \ln \left(\frac{a_{\mathrm{F}}}{a_{\mathrm{in}}}\right)=\frac{1}{p} \ln \left(\frac{\delta_{c}}{\delta_{\psi, \text { in }}}\right) .
$$

with $\delta_{\psi}\left(a_{\mathrm{F}}\right) \equiv \delta_{c} \sim \mathcal{O}(1)$. This number of $e$-folds is typically much smaller than the duration of the RD era. For $\delta_{\psi, \text { in }}$ comparable to $\delta_{R} \approx 10^{-5}$ and $p=(1+\sqrt{5}) / 2$, it only takes $N_{\mathrm{F}} \approx 7 e$-folds before the fluctuations become nonlinear.

We conclude that the fluctuations in the energy density of a nonrelativistic fluid always become nonlinear if the following conditions are satisfied: (i) the coupling $\beta$ is large, (ii) the fraction $\Omega_{\psi}$ reaches a value of the order $\beta^{-2}$ early enough in the $\mathrm{RD}$ era, such that a value $p \approx 1$ is realized, and (iii) the scalar field $\phi$ has a mass smaller than $H$ during the growth period.

An initial $\Omega_{\psi}$ much smaller than $1 /\left(3 \beta^{2}\right)$ grows until it reaches values of the order $1 /\left(3 \beta^{2}\right)$ at $t_{\text {in }}$. During this epoch $p$ is small and the growth of fluctuations remains moderate. Once $\Omega_{\psi}$ reaches the scaling regime, $\Omega_{\psi} \approx 1 /\left(3 \beta^{2}\right)$, the scaling solution with a fast growth of perturbations 12 becomes a good approximation. For small initial $\Omega_{\psi}$ this simply sets the time $t_{\text {in }}$ for the onset of the growth to the time at which $\Omega_{\psi}$ reaches the value $\approx 1 /\left(3 \beta^{2}\right)$. For more detailed considerations one may employ in Eq. 12 a growth rate $p(a)$ that depends on $a$.

\section{BLACK HOLE FORMATION}

When the fluctuations become nonlinear one expects the collapse of overdense regions. A collapsing overdense region of the size of the horizon will presumably form a black hole if nothing stops the approximately spherical infall. An alternative would be the formation of highly concentrated lumps. Black holes interact only by gravitational forces. Thus the $\psi$-particles caught in black holes no longer feel the fifth force. A similar screened behavior can be expected for highly concentrated lumps due to strong backreaction effects [20, 21]. For cosmological purposes black holes and screened objects behave similarly and we will no longer make the distinction.

The $\mathrm{BH}$ formation subtracts from the energy density $\rho_{\psi}$ a fraction $\rho_{\mathrm{BH}}$ that is converted into BHs. Black holes remain stable even if the $\psi$ particles outside them decay at a later stage. The $\mathrm{BH}$ fluid is a nonrelativistic fluid 
contributing to dark matter, with $\rho_{\mathrm{BH}} \sim a^{-3}$ once no new BHs are generated, cf. Fig. 1. A rather small density parameter $\Omega_{\mathrm{BH}}$ generated early in cosmology will grow $\sim a$ during the long RD period and can reach substantial values. During the $\mathrm{BH}$ formation period $\Omega_{\mathrm{BH}}$ will grow even faster than $\sim a$. We neglect here accretion effects after $\mathrm{BH}$ formation which enhance the growth of $\rho_{\mathrm{BH}}$.

There is a simple relation between the time when a black hole forms, as expressed by the scale factor $a_{\mathrm{F}}$ at collapse, and the mass of the black hole $M_{\mathrm{BH}}\left(a_{\mathrm{F}}\right)$. For a rough estimate we assume that all the $\psi$ particles within the horizon at $a_{\mathrm{F}}$ form a $\mathrm{BH}$, with mass

$$
M_{\mathrm{BH}}\left(a_{\mathrm{F}}\right)=\frac{4 \pi}{3} \frac{\rho_{\psi}\left(a_{\mathrm{F}}\right)}{H^{3}\left(a_{\mathrm{F}}\right)} \simeq \frac{4 \pi}{3 \beta^{2}} \frac{M_{P}^{2}}{H_{\mathrm{F}}} .
$$

Here we have employed the value of $\Omega_{\psi}$ according to the scaling solution and $H_{\mathrm{F}} \equiv H\left(a_{\mathrm{F}}\right)$. Actual masses could be somewhat smaller than the estimate (14) if the infalling mass covers only part of the horizon volume. This does not change orders of magnitude. Expressing $M_{\mathrm{BH}}$ in solar mass units $M_{\odot}$ and taking into account the value of the Hubble parameter at matter radiation equality, $H_{\text {eq }}$, we get

$$
\frac{M_{\mathrm{BH}}\left(a_{\mathrm{F}}\right)}{M_{\odot}}=\frac{c}{3 \beta^{2}} \frac{H_{\mathrm{eq}}}{H_{\mathrm{F}}}, \quad c \equiv \frac{M_{\mathrm{eq}}}{M_{\odot}}=2.7 \times 10^{17},
$$

with $M_{\mathrm{eq}}=2 G H_{\mathrm{eq}}^{-1}$. Thus BHs with ten solar masses form at an epoch with $H_{\mathrm{F}} \approx 10^{16} \beta^{-2} H_{\text {eq }}$ or equivalently at a redshift $z_{\mathrm{BH}} \approx 10^{11}|\beta|^{-1}$, typically after nucleosynthesis, $z_{\mathrm{NS}}=10^{9}$. More massive $\mathrm{BHs}$ form even later. We conclude that our mechanism can produce BHs in the mass range observed by the LIGO and VIRGO collaborations only if the mass of the scalar field is below $H_{\mathrm{F}} \approx 2.4 \times 10^{-12} \beta^{-2} \mathrm{eV}$, and if the initial conditions are such that $\Omega_{\psi}$ reaches the scaling solution only near nucleosynthesis.

Let us next estimate the energy density in primordial $\mathrm{BHs}, \rho_{\mathrm{BH}}$. Since $\mathrm{BH}$ formation proceeds very rapidly once the fluctuations $\delta_{\psi}$ become nonlinear, we may assume a complete conversion where all $\psi$ particles end in BHs. In this limit of instantaneous complete conversion one has $\rho_{\mathrm{BH}}\left(a_{\mathrm{F}}\right)=\rho_{\psi}\left(a_{\mathrm{F}}\right)$ and $\Omega_{\mathrm{BH}}\left(a_{\mathrm{F}}\right)=\Omega_{\psi}\left(a_{\mathrm{F}}\right) \approx$ $1 /\left(3 \beta^{2}\right)$. If only a fraction $f$ of the $\psi$ particles ends in black holes our estimate of $\Omega_{\mathrm{BH}}$ has to be multiplied by $f$. After formation $\Omega_{\mathrm{BH}}$ grows like nonrelativistic matter, such that at the end of the RD epoch one has

$$
\Omega_{\mathrm{BH}}\left(a_{\mathrm{eq}}\right)=\frac{a_{\mathrm{eq}}}{a_{\mathrm{F}}} \Omega_{\mathrm{BH}}\left(a_{\mathrm{F}}\right)=\frac{1}{3 \beta^{2}} \frac{a_{\mathrm{eq}}}{a_{\mathrm{F}}} .
$$

For $\Omega_{\mathrm{BH}}\left(a_{\mathrm{eq}}\right)=1 / 2$, the BHs constitute all the dark matter in the Universe. An abundance $\Omega_{\mathrm{BH}}>1 / 2$ would lead to overclosure of the Universe putting bounds on the underlying models with light scalar fields.
Combining Eqs. 15 and 16 we can express the typical mass of the produced BHs in terms of $\beta$ and $\Omega_{\mathrm{BH}}\left(a_{\mathrm{eq}}\right)$,

$$
\frac{M_{\mathrm{BH}}}{M_{\odot}}=\frac{c}{3 \beta^{2}}\left(\frac{a_{\mathrm{F}}}{a_{\mathrm{eq}}}\right)^{2}=\frac{c}{27 \beta^{6} \Omega_{\mathrm{BH}}^{2}\left(a_{\mathrm{eq}}\right)} .
$$

The relations (16) and (17) fix both $M_{\mathrm{BH}} / M_{\odot}$ and $\Omega_{\mathrm{BH}}\left(a_{\mathrm{eq}}\right)$ as functions of the parameters $\beta$ and $a_{\mathrm{F}} / a_{\mathrm{eq}}$. While $\beta$ is a model parameter, $a_{\mathrm{F}} / a_{\text {eq }}$ depends on the initial conditions for $\rho_{\psi}$. For fixed $\beta$, smaller $\rho_{\psi \text {,in leads }}$ to larger $a_{\mathrm{F}} / a_{\mathrm{eq}}$. In particular we may determine the value of $\beta_{c}$ for which dark matter is dominated by $\mathrm{BHs}$

$$
\left|\beta_{c}\right|=585\left(\frac{M_{\mathrm{BH}}}{M_{\odot}}\right)^{-1 / 6}=\left(\frac{2 a_{\mathrm{eq}}}{3 a_{\mathrm{F}}}\right)^{1 / 2} .
$$

For $\beta^{2} \geq 2 a_{\text {eq }} /\left(3 a_{\mathrm{F}}(\beta)\right)$ an additional dark matter component is needed, while models with $\beta^{2} \leq 2 a_{\text {eq }} /\left(3 a_{\mathrm{F}}(\beta)\right)$ are excluded.

We finally relate $a_{\mathrm{F}} / a_{\mathrm{eq}}$ to the initial conditions for $\Omega_{\psi}$ in different scenarios:

1. In our first scenario the $\psi$ particles are produced during the heating and entropy production after inflation. We will denote the end of the heating period by $a_{\mathrm{ht}}$. If $\Omega_{\psi}\left(a_{\mathrm{ht}}\right)$ is of the order $1 /\left(3 \beta^{2}\right)$ the fluctuations become nonlinear at $a_{\mathrm{F}} / a_{\mathrm{ht}}=\exp \left(N_{\mathrm{F}}\right)$, or equivalently at $a_{\mathrm{F}} / a_{\mathrm{eq}}=\exp \left(N_{\mathrm{F}}-N_{\text {eq }}\right)$ with $N_{\text {eq }}$ the number of $e-$ folds between the end of the heating period and matterradiation equality. If we want to prevent the dimensionless coupling $g$ from being larger than one, $\beta^{2}$ is bounded by $M_{P}^{2} / m^{2}$. Since $m^{2}$ must be larger than $H^{2}\left(a_{\mathrm{ht}}\right)$, this puts a limit on the end of the heating period,

$$
N_{\mathrm{eq}} \lesssim \ln \left(\frac{M_{P}}{H_{\mathrm{eq}}}\right)^{2 / 5}+\frac{N_{\mathrm{F}}}{5} \simeq 50+\frac{N_{\mathrm{F}}}{5} .
$$

The critical value $\beta_{c}$ for $\mathrm{BH}$ dark matter is very large in this scenario,

$$
\beta_{c} \approx \exp \left[\left(N_{\mathrm{eq}}-N_{\mathrm{F}}\right) / 2\right]
$$

and according to Eq. (17) typical BH masses are tiny as compared to the solar mass. For $\Omega_{\psi}\left(a_{\mathrm{ht}}\right)$ larger than $1 /\left(3 \beta^{2}\right)$ the growth of fluctuations will be even faster, with smaller $N_{\mathrm{F}}$ and $\mathrm{BH}$ masses.

2. For a second scenario we assume $\Omega_{\psi}\left(a_{\mathrm{ht}}\right) \ll$ $1 /\left(3 \beta^{2}\right)$, while the $\psi$ particles are already decoupled from radiation. In this case the onset of growth is delayed by the time $\Omega_{\psi}$ needs to grow to the order $1 /\left(3 \beta^{2}\right)$. This replaces $N_{\text {eq }} \rightarrow N_{\text {eq }}+\ln \left(3 \beta^{2} \Omega_{\psi}\left(a_{\text {ht }}\right)\right)$ in Eqs. (19) and 20. As can be seen from Fig. 1 the relation between $\rho_{\psi}$ before the scaling solution and $\Omega_{\mathrm{BH}}\left(a_{\mathrm{eq}}\right)$ is almost independent of $\beta$.

3. In a third scenario the $\psi$ particles are in thermal equilibrium at $a_{\mathrm{ht}}$. They decouple from radiation at some time $a_{\mathrm{dc}}$. At this time, their abundance can be strongly Boltzmann suppressed, such that $\Omega_{\psi}\left(a_{\mathrm{dc}}\right)$ can be very 
small. Effectively, this replaces in Eqs. 19 and 20 $N_{\text {eq }} \rightarrow N_{\text {eq }}+\ln \left(3 \beta^{2} \Omega_{\psi}\left(a_{\mathrm{dc}}\right) a_{\mathrm{ht}} / a_{\mathrm{dc}}\right)$. The BH masses can now be substantially larger, and the LIGO and VIRGO range can be realized for sufficiently small $\Omega_{\psi}\left(a_{\mathrm{dc}}\right)$.

\section{CONCLUSIONS}

In this paper we presented a new mechanism for black hole formation which does not rely on inflationary physics. We argued that primordial black holes could be generated by long-range interactions as those ubiquitously appearing in beyond the Standard Model scenarios or modified gravity/dark energy theories. For illustration purposes we considered a very simple scenario containing only a light scalar field coupled with some fermion field beyond the Standard Model. We showed that for sufficiently large couplings, the system enters a scaling regime in which the fermion energy density tracks the background component. During this regime the primordial fermion perturbations become significantly enhanced and can eventually collapse into black holes.

Can the produced black holes contribute to dark matter? There are tight observational constraints on the abundance of PBHs [22]. According to Refs. [7, 23], there exists an open window for dark matter BHs around 1$1000 M_{\odot}$ (see also Refs. [24, 25]). Since this is also the interesting regime to explain the gravitational wave detections, we will focus next on models for which the $\mathrm{BH}$ mass distribution peaks at $M_{\max } \sim \mathcal{O}\left(M_{\odot}\right)$. We emphasize, however, that this choice is made for illustration purposes only and that other ranges of masses can be easily accommodated by different choices of $\beta$. The value of $M_{\text {max }}$ depends indeed on the precise value of this effective coupling. For $\mathrm{BH}$ dark matter $\left[\Omega_{\mathrm{BH}}\left(a_{\mathrm{eq}}\right)=1 / 2\right]$ it is well approximated by Eq. (18),

$$
M_{\max } \simeq\left(\frac{585}{\beta}\right)^{6} M_{\odot} .
$$

A more refined analysis taking into account the entropy production between $a_{\mathrm{F}}$ and $a_{\mathrm{eq}}$, as well as the distribution of $\delta_{\psi}$ perturbations via a Press-Schechtertype formalism [26], changes the maximum mass 21] by an $\mathcal{O}(1)$ multiplicative factor $\Delta g_{s}(p /(2+p))^{1 / p}$, with $\Delta g_{s}=\left(g_{s}\left(a_{\mathrm{eq}}\right) / g_{s}\left(a_{\mathrm{F}}\right)\right)^{1 / 2}$ and $g_{s}$ the number of entropic degrees of freedom. The mean and the standard deviation of the $\mathrm{BH}$ distribution are very close to $M_{\max }$, leading to a nearly monochromatic mass spectrum. To obtain masses in the range $1-1000 M_{\odot}$, we must have couplings in the range $185 \lesssim \beta \lesssim 585$. For the corresponding very small density parameter in the scaling regime, $\Omega_{\psi}=1 /\left(3 \beta^{2}\right)$, there are no constraints from nucleosynthesis. Black hole formation takes place at an epoch $a_{\mathrm{F}}=2 a_{\mathrm{eq}} /\left(3 \beta^{2}\right)$, cf. Eq. (18), corresponding to temperatures of order $\mathcal{O}(\mathrm{MeV})$. The $\psi$-particles must be stable until this epoch.
Our results are only rough order of magnitude estimates. A more detailed account of the formation and evolution processes is needed. On the one hand, merging and accretion effects will tend to shift and broaden the $\mathrm{BH}$ mass distribution [27, 28]. On the other hand, extensions and modifications of the model with more than one heavy particles' species may lead to nonmonocromatic spectra and modifications of the observational constraints [25]. In view of the present uncertainties, a production of black holes in the range $1-1000 M_{\odot}$ and constituting the whole dark matter component of the Universe seems possible. The necessary ingredients are a scalar field with mass smaller than $10^{-14} \mathrm{eV}$, heavy fields in a suitable abundance, and a mutual effective coupling of the order $\beta_{c}$. The almost massless scalar field could be the cosmon of dynamical dark energy [12, 29, 30, which has at all times a dynamical mass of the order $H$.

\section{ACKNOWLEDGEMENTS}

We acknowledge support from the DFG through the project TRR33, "The Dark Universe".

* l.amendola@thphys.uni-heidelberg.de

$\dagger$ j.rubio@thphys.uni-heidelberg.de

c.wetterich@thphys.uni-heidelberg.de

[1] Y. B. Zel'dovich, I. D. Novikov, Astron. Zh. 43, 758, (1966)

[2] S. Hawking, Mon. Not. Roy. Astron. Soc. 152 (1971) 75.

[3] B. J. Carr, Astrophys. J. 201 (1975) 1.

[4] George F. Chapline, Nature 253, 251-252, 1975.

[5] B. P. Abbott et al. [LIGO Scientific and Virgo Collaborations], Phys. Rev. Lett. 116 (2016) no.6, 061102; B. P. Abbott et al. [LIGO Scientific and Virgo Collaborations], Phys. Rev. Lett. 116 (2016) no.24, 241103; B. P. Abbott et al. [LIGO Scientific and VIRGO Collaborations], Phys. Rev. Lett. 116, no. 20, 201301 (2016); S. Bird, I. Cholis, J. B. Muñoz, Y. Ali-Haïmoud, M. Kamionkowski, E. D. Kovetz, A. Raccanelli and A. G. Riess, Phys. Rev. Lett. 116 (2016) no.20, 201301; S. Blinnikov, A. Dolgov, N. K. Porayko and K. Postnov, JCAP 1611 (2016) no.11, 036.

[6] M. Kawasaki, A. Kusenko and T. T. Yanagida, Phys. Lett. B 711 (2012) 1

[7] B. Carr, F. Kuhnel and M. Sandstad, Phys. Rev. D 94 (2016) no.8, 083504

[8] J. Garcia-Bellido, A. D. Linde and D. Wands, Phys. Rev. D 54 (1996) 6040; J. Yokoyama, Astron. Astrophys. 318 (1997) 673; T. Nakamura, M. Sasaki, T. Tanaka and K. S. Thorne, Astrophys. J. 487 (1997) L139; M. Drees and E. Erfani, JCAP 1104 (2011) 005; M. Kawasaki, A. Kusenko, Y. Tada and T. T. Yanagida, Phys. Rev. D 94 (2016) no.8, 083523; J. Garcia-Bellido and E. Ruiz Morales, Phys. Dark Univ. 18 (2017) 47; K. Kannike, L. Marzola, M. Raidal and H. Veermäe, JCAP 1709 (2017) no.09, 020; C. Germani and T. Prokopec, Phys. 
Dark Univ. 18 (2017) 6; H. Motohashi and W. Hu, Phys. Rev. D 96 (2017) no.6, 063503.; G. Ballesteros and M. Taoso, Phys. Rev. D 97 (2018) no.2, 023501.

[9] M. Crawford and D. N. Schramm, Nature 298 (1982) 538; S. W. Hawking, I. G. Moss and J. M. Stewart, Phys. Rev. D 26 (1982) 2681; H. Kodama, M. Sasaki and K. Sato, Prog. Theor. Phys. 68 (1982) 1979; D. La and P. J. Steinhardt, Phys. Lett. B 220 (1989) 375; I. G. Moss, Phys. Rev. D 50 (1994) 676; R. V. Konoplich, S. G. Rubin, A. S. Sakharov and M. Y. Khlopov, Phys. Atom. Nucl. 62 (1999) 1593; C. J. Hogan, Phys. Lett. 143B (1984) 87; S. W. Hawking, Phys. Lett. B 231 (1989) 237; A. Polnarev and R. Zembowicz, Phys. Rev. D 43 (1991) 1106; R. R. Caldwell and P. Casper, Phys. Rev. D 53 (1996) 3002; H. B. Cheng and X. Z. Li, Chin. Phys. Lett. 13 (1996) 317; J. H. MacGibbon, R. H. Brandenberger and U. F. Wichoski, Phys. Rev. D 57 (1998) 2158; V. A. Berezin, V. A. Kuzmin and I. I. Tkachev, Phys. Lett. 120B (1983) 91; R. R. Caldwell, A. Chamblin and G. W. Gibbons, Phys. Rev. D 53 (1996) 7103.

[10] E. Cotner and A. Kusenko, Phys. Rev. Lett. 119 (2017) no.3, 031103; E. Cotner and A. Kusenko, Phys. Rev. D 96 (2017) no.10, 103002.

[11] M. Magg and C. Wetterich, Phys. Lett. 94B (1980) 61.

[12] C. Wetterich, Astron. Astrophys. 301 (1995) 321

[13] C. Wetterich, Phys. Lett. B 655 (2007) 201

[14] L. Amendola, M. Baldi and C. Wetterich, Phys. Rev. D 78 (2008) 023015

[15] R. Fardon, A. E. Nelson and N. Weiner, JCAP 0410 (2004) 005

[16] A. W. Brookfield, C. van de Bruck, D. F. Mota and D. Tocchini-Valentini, Phys. Rev. D 73 (2006) 083515 Erratum: [Phys. Rev. D 76 (2007) 049901]

[17] S. Casas, V. Pettorino and C. Wetterich, Phys. Rev. D 94 (2016) no.10, 103518
[18] L. Amendola, Phys. Rev. D 62 (2000) 043511

[19] L. Amendola, Phys. Rev. D 69 (2004) 103524 doi:10.1103/PhysRevD.69.103524

[20] Y. Ayaita, M. Weber and C. Wetterich, Phys. Rev. D 87 (2013) no.4, 043519 doi:10.1103/PhysRevD.87.043519

[21] Y. Ayaita, M. Weber and C. Wetterich, Phys. Rev. D 85 (2012) 123010 doi:10.1103/PhysRevD.85.123010

[22] P. Tisserand et al. [EROS-2 Collaboration], Astron. Astrophys. 469 (2007) 387; M. Ricotti, J. P. Ostriker and K. J. Mack, Astrophys. J. 680 (2008) 829; L Wyrzykowski et al., Mon. Not. Roy. Astron. Soc. 458 (2016) no.3, 3012; E. Mediavilla, J. JiménezVicente, J. A. Muñoz, H. Vives-Arias and J. CalderónInfante, Astrophys. J. 836 (2017) no.2, L18; V. Poulin, P. D. Serpico, F. Calore, S. Clesse and K. Kohri, Phys. Rev. D 96 (2017) no.8, 083524; Y. Ali-Haïmoud and M. Kamionkowski, Phys. Rev. D 95 (2017) no.4, 043534; T. D. Brandt, Astrophys. J. 824 (2016) no.2, L31; A. M. Green, Phys. Rev. D 94 (2016) no.6, 063530; T. S. Li et al. [DES Collaboration], Astrophys. J. 838 (2017) no.1, 8.; Y. Inoue and A. Kusenko, JCAP 1710 (2017) no.10, 034.

[23] S. Clesse and J. García-Bellido, arXiv:1711.10458 [astroph.CO].

[24] F. Kühnel and K. Freese, Phys. Rev. D 95 (2017) no.8, 083508

[25] B. Carr, M. Raidal, T. Tenkanen, V. Vaskonen and H. Veermäe, Phys. Rev. D 96 (2017) no.2, 023514

[26] W. H. Press and P. Schechter, Astrophys. J. 187 (1974) 425.

[27] J. R. Chisholm, Phys. Rev. D 73 (2006) 083504

[28] B. J. Carr, K. Kohri, Y. Sendouda and J. Yokoyama, Phys. Rev. D 81 (2010) 104019

[29] C. Wetterich, Nucl. Phys. B 302 (1988) 668

[30] J. Rubio and C. Wetterich, Phys. Rev. D 96 (2017) no.6, 063509 